\title{
Hybrid Field Orientation and Direct Torque Control for Electric Vehicle Motor Drive with an Extended Kalman Filter
}

\author{
Mehdi Farasat, Ekrem Karaman, Andrzej M. Trzynadlowski, Fellow, IEEE, \\ and M. Sami Fadali, Senior Member, IEEE
}

\begin{abstract}
An electric vehicle (EV) drive must feature fast torque response, high efficiency over wide speed and torque ranges, and reasonable cost. This paper proposes an efficient and robust control scheme for a speed sensorless EV with an induction motor. The main control strategy is a hybrid field oriented control (FOC) and direct torque control (DTC) which combines the advantages of both FOC and DTC and eliminates certain implementation difficulties. For robust operation above the rated speed, a control scheme for field weakening is developed. Since sensorless control is a lower-cost alternative to the position or speed encoder-based control, an extended Kalman filter based speed estimation method is adopted in the main control strategy. Furthermore, a loss-model-based controller (LMC) is employed to optimize the efficiency of the drive. Simulation results verify the effectiveness of the proposed method.
\end{abstract}

Index Terms-Electric vehicle, extended kalman filter, field weakening, induction motor, loss minimization

\section{INTRODUCTION}

$\mathrm{T}$ The development of electric vehicles (EVs) has taken on an accelerated pace for mass marketing with everincreasing concerns about energy efficiency and environmental protection. The performance of EVs has improved and they are gradually becoming a viable alternative to vehicles with internal combustion engines (ICEs). However, EVs still have a far shorter driving range than traditional vehicles. From the viewpoint of control engineering, the control system is the key for improving motor efficiency, lengthening battery life, and consequently increasing the driving range. However, because the motor parameters and the operating conditions vary during EV operation, this control task is not easy to accomplish with traditional controllers [1].

Selection of the traction motor for an EV propulsion system is a crucial step in EV design. Many criteria such as efficiency, cost, reliability, power density, maturity of technology and controllability must be taken into consideration. The most commonly used motors in hybrid electric vehicles (HEV) and pure electric vehicles (PEV) are: $\mathrm{DC}$, induction, permanent magnet synchronous, switched reluctance and brushless DC motors [2]. A comparison of these motors concluded that the induction motor (IM) is the best candidate for EV applications due to its robustness, low price, mature technology, and maintenance-free operation [2].

The authors are with the Department of Electrical and Biomedical Engineering, University of Nevada, Reno, NV 89557-0260 USA (emails: mfarasat@unr.edu, ekaraman@unr.edu, chin@unr.edu, fadali@unr.edu )

U.S. Government work not protected by U.S. copyright
Consequently, a squirrel-cage IM is chosen for the drive system considered in this paper.

Although many IM control techniques have been proposed, the two most popular methods are field oriented control (FOC) and direct torque control (DTC). Unlike FOC, DTC does not require current controllers, coordinate transformation or PWM signal generators. In spite of its simplicity, DTC provides good torque control in the steady state and under transient operating conditions. In addition, this control is highly insensitive to parameter detuning. On the other hand, DTC has significant disadvantages including high current and torque ripple, and lack of direct current control [3]. The recently introduced Hybrid FOC-DTC method [4] enjoys the advantages of both FOC and DTC and alleviates their implementation difficulties. Since an EV drive system must feature a fast torque response, reasonable cost, reliability, and robustness, the hybrid method appears to be particularly suited to EV applications.

From the drive system point of view, eliminating speed sensors and the associated auxiliaries has the advantage of lower cost, robustness, and increased reliability. Traditional approaches to speed sensorless control employ flux and slip estimation using stator currents and voltages, but cause large speed estimation errors, particularly in the low-speed range. Model reference adaptive system (MRAS) technology has also been used to estimate the speed of an IM. This approach also results in large estimation errors in the low-speed range and in large steady-state errors.

In addition to the aforementioned studies, the extended Kalman filter (EKF) has also been proposed in the literature as a stochastic approach to motor speed estimation. The EKF is known for its high convergence rate, which improves transient performance significantly. Additionally, accurate estimation and convergence in the steady state require high-frequency signals, which are also inherently satisfied by EKFs with the model and measurement noises included in the model [5]. In this paper, an EKF-based estimation algorithm is developed for use with the speed sensorless hybrid FOC-DTC of an IM propelling the vehicle.

In EV applications, electric drive must operate with high efficiency over a wide speed range. At low speeds, the drive delivers constant torque, whereas a constant power with speed related torque is requested at the medium and high speeds. Operation in the constant power region is attained by field weakening. Conventionally, in the case of IM drives, the flux 
reference is fixed at the rated value for speeds below the base speed, and is reduced in inverse proportion to the speed for higher speeds. However, this is not an optimal solution [6]. In this paper, a control scheme for robust field weakening operation of motor drive is adopted. The basic idea is to adjust the flux reference on the basis of the q-axis stator current error, thus determining spontaneous field weakening.

As concluded in [7], compared to permanent magnet motors, IMs have comparable energy efficiencies under standard driving schedules when their control system employs loss minimization algorithms. In this paper a loss-model-based controller (LMC) is used to determine an optimum flux level for the efficiency optimization of the IM drive. The suggested LMC is simple, but leakage inductances are not neglected in order to determine an accurate loss equation.

\section{CONTROL STRATEGY}

It has been demonstrated in [4] that FOC and DTC share a common basis despite their implementation differences. Regarding the FOC at constant flux, it can be shown that

$\left\{\begin{array}{c}\left|\vec{\lambda}_{r}\right|=k_{d} i_{d s} \\ T_{e}=k_{q} i_{q s}\end{array} \Rightarrow\left\{\begin{array}{c}\left|\vec{\lambda}_{r}\right| \propto i_{d s} \\ T_{e} \propto i_{q s}\end{array} \Rightarrow\left\{\begin{array}{c}\Delta\left|\vec{\lambda}_{r}\right| \propto \Delta i_{d s} \\ \Delta T_{e} \propto \Delta i_{q s}\end{array}\right.\right.\right.$

where $k_{d}$ and $k_{q}$ depend on motor parameters and $\Delta$ denotes small variations. $i_{d s}, i_{q s}$ are the $d-q$ axis stator currents, $\lambda_{r}$ and $T_{e}$ are rotor flux and electromagnetic torque, respectively. Also,

$$
\Delta T_{e} \propto \Delta \lambda_{T}
$$

where $\lambda_{T}$ is the tangential component of the stator flux linkage vector. Recalling the basic theory of DTC, it is apparent that the variation in the stator flux magnitude is

$$
\Delta\left|\vec{\lambda}_{s}\right|=\Delta \lambda_{F}
$$

where $\lambda_{F}$ is the radial component of the stator flux linkage vector. If the first order delay between $\lambda_{r}$ and $\lambda_{s}$ is ignored, (3) yields

$$
\Delta\left|\vec{\lambda}_{r}\right|=\Delta \lambda_{F}
$$

Comparing (2) and (4) to (1) yields

$$
\left\{\begin{array}{l}
\Delta \lambda_{F} \propto \Delta i_{d s} \\
\Delta \lambda_{T} \propto \Delta i_{q s}
\end{array}\right.
$$

Thus, the radial component of stator flux linkage variation in DTC is proportional to the $d$-axis component of stator current vector variation in $\mathrm{FOC}$, and both quantities are involved in motor flux control. Also, the tangential component of the stator flux linkage variation in DTC is proportional to the $q$-axis stator current vector variation in FOC and both affect the control of the motor torque. Thus, there is a direct relationship between the hysteresis control of flux linkage in DTC and d-axis current control in FOC. Also, the hysteresis control of electromagnetic torque in DTC corresponds closely to the q-axis current control in FOC. Consequently, a motor drive control system, having $d$ - and $q$-axis current hysteresis controllers as in FOC, and a switching table as in DTC, has been proposed in [4]. Fig.1 depicts the block diagram of a hybrid field oriented and direct torque controlled induction motor drive.

\section{FIELD WEAKENING}

In many applications, the electric drive has to deliver a constant torque at low speeds, whereas a constant power is required at medium and high speeds. The constant-power region is obtained by field weakening. The choice of flux reference and the base speed corresponding to the beginning of the field weakening region is very critical. It is known that setting the flux reference level at lower than the optimal level causes nearly proportional reduction of the torque capability due to the inverter current limit. On the other hand, choosing higher than optimal flux reference levels cause an increase in the back EMF. As a consequence, for a prefixed dc-link voltage the maximum current becomes unavailable. This in turn decreases the torque production capability of the motor.

In this paper, field weakening is implemented by monitoring the motor behavior for $N$ consecutive cycles. If the output $k_{q}$ of the $q$-axis stator current hysteresis comparator remains one during this time interval, only active vectors are applied to the motor and the torque demand is not satisfied. The control algorithm assumes that the selected flux level is higher than the required level for the actual operating conditions and a small reference flux reduction $\Delta \lambda_{r}=-\delta$ is applied. If the control algorithm applies at least one zero or inverse configuration during the $N$ cycle periods, the algorithm assumes that the torque demand is satisfied, and the control algorithm attempts to increase the flux reference.

The main goal of this control algorithm is to determine the maximum flux level that ensures that the torque command is realized. The flux cannot become lower than a minimum value $\lambda_{r, \min }$ or greater than the rated value $\lambda_{r, \text { rated }}$. These basic principles ensure correct control of the flux reference over the whole speed range, and a smooth transition between the constant flux and field weakening regions, with no need for flux reference calculations or accurate knowledge of motor parameters. Independent from the machine parameters, this scheme self-regulates the rotor flux reference, provides satisfactory robust operation over the entire speed range. 


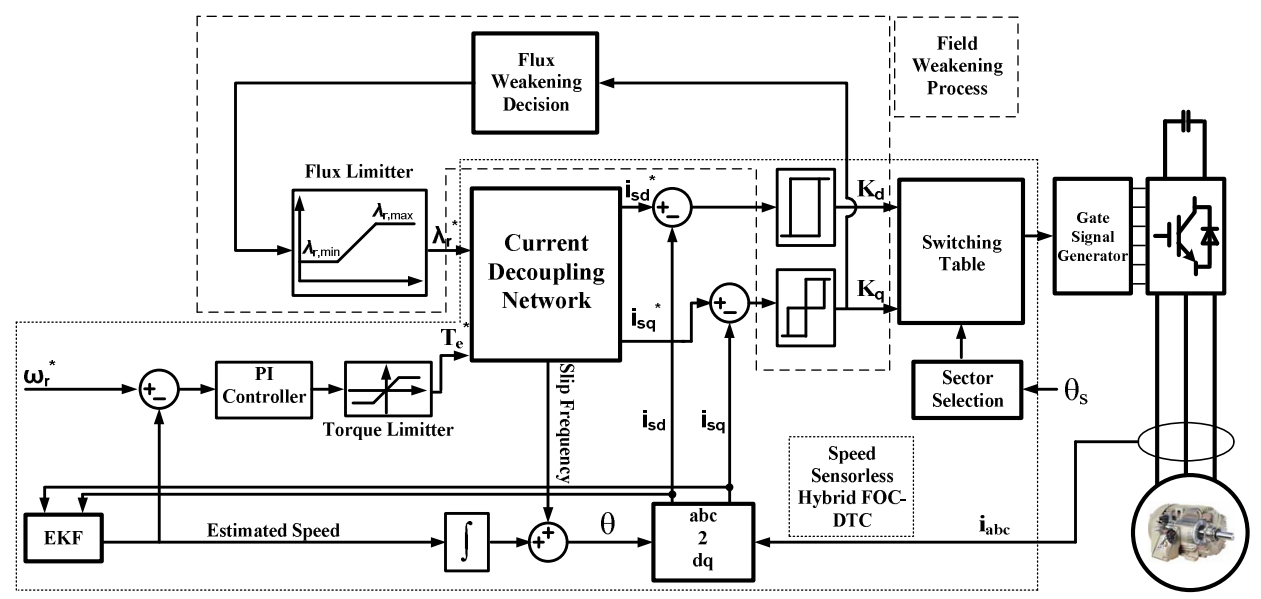

Fig. 1. Speed sensorless Hybrid FOC-DTC scheme with field weakening

\section{LOSS-MINIMIZATION ALGORITHM}

In the development of the loss model, the induction motor equivalent circuit in the synchronous reference frame is used. The equivalent circuit is shown in Fig. 2.
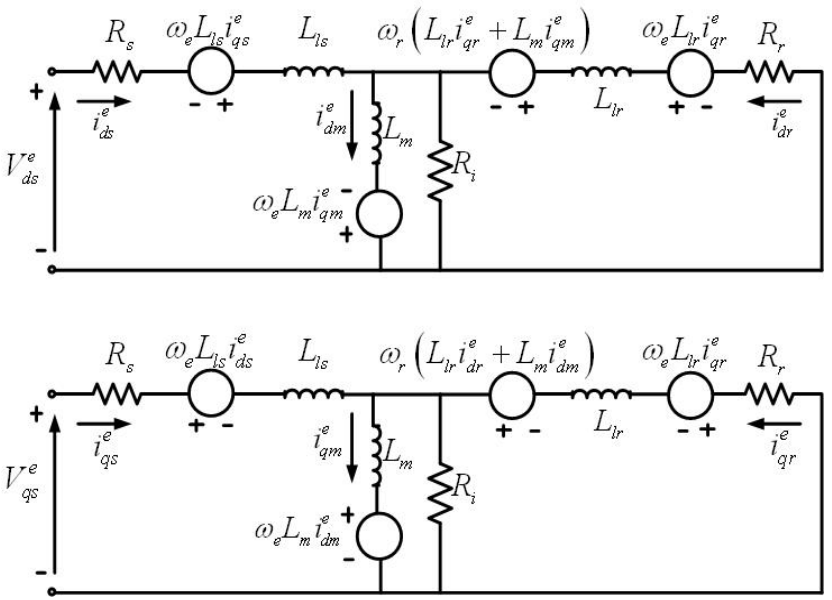

Fig. 2. Induction motor equivalent circuit in the synchronous reference frame

A problem with the previous model is the complexity caused by the current flowing through the iron loss branch $\left(R_{i}\right)$. To simplify the model, the leakage inductances are ignored. This simplification is effective in low-frequency operations but is inappropriate for high electrical angular velocity $\omega_{e}$ where the voltage drop across the leakage inductance becomes significant.

In this paper, model simplification is based on the fact that the magnetizing currents $i_{d m}, i_{q m}$ flowing through the magnetizing inductance $\left(L_{m}\right)$ are much larger than the corresponding loss current flowing through the parallel iron loss branch [8].

There are four types of major losses in an IM: (1) copper loss in the stator; (2) core loss in the stator; (3) copper loss in the rotor; and (4) core loss in the rotor. Core loss in the rotor can be neglected, because at the rated speed the slip frequency is small. In the optimum flux calculation, the inverter losses can also be ignored for low-power IM drives [9]. Since the $d$ axis stator current $\left(i_{d s}\right)$ is responsible for motor flux control of, the optimum flux level is obtained by equating the derivative of the total losses with respect to $i_{d s}$ to zero [10].

$$
\frac{\partial P_{\text {loss }}}{\partial i_{d s}}=0 \Rightarrow i_{d s}^{*}=\sqrt{\frac{\left(R_{S}+\omega_{e}^{2} L_{m}^{2}\right)}{\left(R_{S}+\frac{R_{r} L_{m}^{2}}{L_{r}^{2}}+\frac{\omega_{e}^{2}\left(L_{m}^{2}-L_{S} L_{r}\right)^{2}}{R_{i} L_{r}^{2}}\right)}} i_{q s}
$$

Then, the optimal flux level is given by

$$
\lambda_{d r}^{*}=L_{m} i_{d s}^{*}
$$

\section{SPEED ESTIMATION}

A dynamic electrical model for a three-phase IM has four state variables, namely, the stator currents $\left(i_{d s}, i_{q s}\right)$ and the rotor flux $\left(\lambda_{d r}, \lambda_{q r}\right)$. An extended IM model results if the rotor speed is included as an additional state variable. The EKFbased estimators are based on the extended IM model in the following general form

$$
\begin{aligned}
\underline{\dot{x}}_{e}(t) & =\underline{f}_{e}\left(\underline{x}_{e}(t), \underline{u}_{e}(t)\right)+G \underline{w}(t) \\
& =\underline{A}_{e}\left(\underline{x}_{e}(t)\right) \underline{x}_{e}(t)+\underline{B}_{e} \underline{u}_{e}(t)+G \underline{w}(t) \\
\underline{Z}(t) & =\underline{h}_{e}\left(\underline{x}_{e}(t)\right)+\underline{v}(t) \\
& =\underline{H}_{e} \underline{x}_{e}(t)+\underline{v}(t)
\end{aligned}
$$

Here, the extended state vector $\underline{x}_{e}$ represents the estimated states, $\underline{f}_{e}$ : nonlinear function of the states and inputs, $\underline{A}_{e}$ : system matrix, $\underline{u}_{e}$ : control input vector, $\underline{B}_{e}$ : input matrix, $\underline{h}_{e}$ : function of the outputs, $\underline{H}_{e}$ : measurement matrix, $G$ :noise weight matrix, $\underline{w}(t)$ : process noise, $\underline{v}(t)$ : measurement noise. The covariance matrices $Q$ and $R$ of these noises are defined as

$$
\begin{gathered}
Q=\operatorname{cov}(w)=E\left\{w w^{T}\right\} \\
R=\operatorname{cov}(v)=E\left\{v v^{T}\right\}
\end{gathered}
$$

and based on the general form in (8) and (9), the extended model of IM based on the stator flux is given by (10) and (11).

An EKF algorithm has been developed for the estimation of states in the extended IM model given by (10) and (11) to 
be used in the sensorless control of the IM. The KF method used for this purpose is a recursive algorithm. It takes the stochastic state space model of the system into account, together with the measured outputs, to achieve the optimal estimation of states in multi-input multi-output systems. The system and measurement noises are considered to be in the form of zero-mean Gaussian white noise. The optimality of the state estimation is achieved by minimizing the covariance of the estimation error. For nonlinear problems the KF is not strictly applicable since linearity plays an important role in its derivation and performance as an optimal filter. EKF theory attempts to overcome this difficulty by a linearized approximation approach where the linearization is performed on the current state estimate [5]. This process requires the discretization of (10) and (11) as shown below

$$
\begin{aligned}
x_{e}(k+1) & =\underline{f}_{e}\left(\underline{x}_{e}(k), \underline{u}_{e}(k)\right)+G \underline{w}(k) \\
\underline{z}(k) & =\underline{H}_{e} \underline{x}_{e}(k)+\underline{v}(k)
\end{aligned}
$$

As mentioned before, the EKF involves the linearized approximation of the nonlinear model (11) and (12) and uses current estimation of states $\hat{x}_{e}(k)$ in linearization by using

$$
\underline{F}_{e}(k)=\frac{\partial f_{e}\left(x_{e}(k), u_{e}(k)\right)}{\partial x_{e}(k)}
$$

The EKF algorithm is illustrated in Fig.3. It involves two main stages: prediction and correction. In the prediction stage, the next predicted states and predicted state error covariance matrices are processed, while in the correction stage, the next estimated states, obtained as the sum of the next predicted states and the correction term, are calculated.

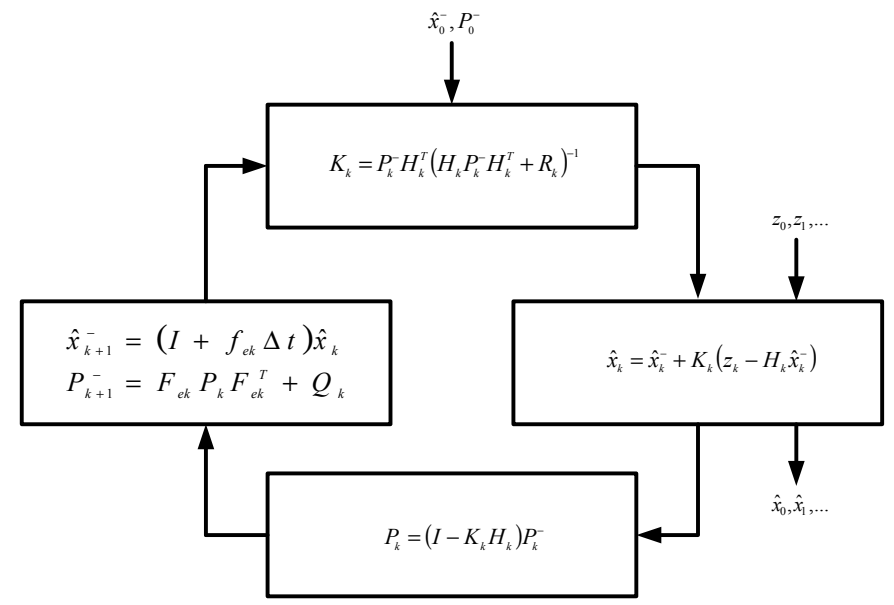

VI. SIMULATION RESULTS

The parameters of the simulated $1.5 \mathrm{~kW}$ induction motor are listed in Table I. The error covariance matrix $P$ of the EKF was initially set as an identity matrix, while the noise covariance matrices $R, Q$ and noise weight matrix $G$ were assumed as

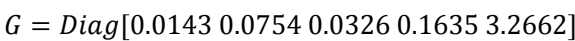

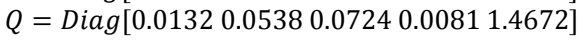

$$
\begin{aligned}
& R=\operatorname{Diag}[0.0163 \text { 0.0085] }
\end{aligned}
$$

To evaluate the performance of the developed control system for the EV IM drive, four cases were considered with various torque and speed references and motor parameters.

\section{Case 1- Low Speed}

For the motor parameters of Table I, the rated speed of the motor is around $300 \mathrm{rad} / \mathrm{sec}$. This case is to verify the performance of the EKF-based speed estimation method at low speeds. To do so, the motor is accelerated up to 30 $\mathrm{rad} / \mathrm{sec}$. After reaching the reference speed at $t=0.5 \mathrm{sec}$, speed is reduced down to $10 \mathrm{rad} / \mathrm{sec}$ at $t=1 \mathrm{sec}$ and is kept at this level for $0.5 \mathrm{sec}$. The load torque of $10 \mathrm{Nm}$ is applied at $t=0.6$ $\mathrm{sec}$ and removed at $t=0.9 \mathrm{sec}$. Another step change in the load torque $(5 \mathrm{Nm})$ is applied during the 1.6-1.9 sec time interval. The simulation results are shown in Fig. 4. The errors obtained in the speed estimate are mainly due to uncertainties in the stator inductance and resistance.

\section{Case 2- High Speed}

Fig. 5 shows the performance of the drive in the field weakening region. In this case, the motor is accelerated from $200 \mathrm{rad} / \mathrm{sec}$ to $500 \mathrm{rad} / \mathrm{sec}$ at $t=0.7 \mathrm{sec}$ and decelerated to 0 $\mathrm{rad} / \mathrm{sec}$ at $t=1.6 \mathrm{sec}$. A load torque of $2 \mathrm{Nm}$ is applied at $t$ $=1.3 \mathrm{sec}$ and removed at $t=1.5 \mathrm{sec}$. The rotor flux amplitude is automatically reduced when the speed command is above the base speed. In the field weakening region, the motor drive is designed to draw the maximum permissible current in order to produce the maximum torque at the reduced flux.

\section{Case 3-Efficiency Optimization at Steady-State}

The results of flux optimization during the steady-state operation with $60 \pi \mathrm{rad} / \mathrm{sec}$ reference speed and a $2 \mathrm{Nm}$ load torque are shown in Fig. 6. To show the effectiveness and dynamic performance of the optimization method, the following simulation strategy was adopted: 1) A nominal flux of $0.6 \mathrm{~Wb}$ is applied to the induction motor drive until it reaches its steady state; 2 ) at $t=0.9 \mathrm{sec}$ the loss-minimization strategy is activated.

Fig.3. Extended Kalman filter loop

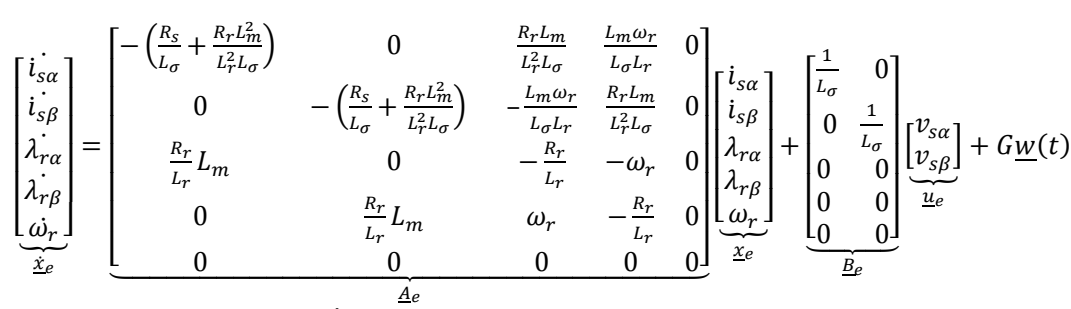

$$
\begin{aligned}
& \underbrace{\left[\begin{array}{c}
i_{s \alpha} \\
i_{s \beta}
\end{array}\right]}_{\underline{\underline{a}}}=\underbrace{\left[\begin{array}{lllll}
1 & 0 & 0 & 0 & 0 \\
0 & 1 & 0 & 0 & 0
\end{array}\right]}_{\underline{H}_{e}}\left[\begin{array}{c}
i_{s \alpha} \\
i_{s \beta} \\
\lambda_{r \alpha} \\
\lambda_{r \beta} \\
\omega_{r}
\end{array}\right]+\underline{v}(t)
\end{aligned}
$$


Table I

\begin{tabular}{cccc}
\hline \multicolumn{4}{c}{ Specifications of the Simulated Induction Motor } \\
$\mathrm{f}[\mathrm{Hz}]$ & $\mathrm{R}_{\mathrm{s}}[\Omega]$ & $\mathrm{R}_{\mathrm{r}}[\Omega]$ & $\mathrm{R}_{\mathrm{i}}[\Omega]$ \\
50 & 0.5 & 1 & 200 \\
$\mathrm{~L}_{\mathrm{s}}=\mathrm{L}_{\mathrm{r}}[\mathrm{H}]$ & $\mathrm{L}_{\mathrm{m}}[\mathrm{H}]$ & $\mathrm{p}$ & $\mathrm{J}\left[\mathrm{kg} \cdot \mathrm{m}^{2}\right]$ \\
0.105 & 0.1 & 2 & 0.01 \\
\hline
\end{tabular}

Flux optimization during steady-state operation can considerably reduce the flux amplitude and input currents of the motor. This improves the efficiency of the motor.

Case 4- Stator and Rotor Resistances Variations under EKF

In this case, the performance of the EKF is evaluated under $R_{s}$ and $R_{r}$ variation. The motor is accelerated to $60 \pi \mathrm{rad} / \mathrm{sec}$ with different values of the stator and rotor resistances. The simulation was carried out for four cases with values of 1. $\dot{R}_{s}=R_{s}, R_{r}=R_{r} ; 2 . \dot{R}_{s}=1.5 \times R_{s}, R_{r}=R_{r} ; 3 . \dot{R}_{s}=R_{s}$, $R_{r}=1.5 \times R_{r} ; 4$. $R_{s}=1.5 \times R_{s}, R_{r}=1.5 \times R_{r}$. The results shown in Fig. 7 show that the estimation error obtained when only one of the resistances value is incorrect, is significantly lower than that for errors in both resistances.

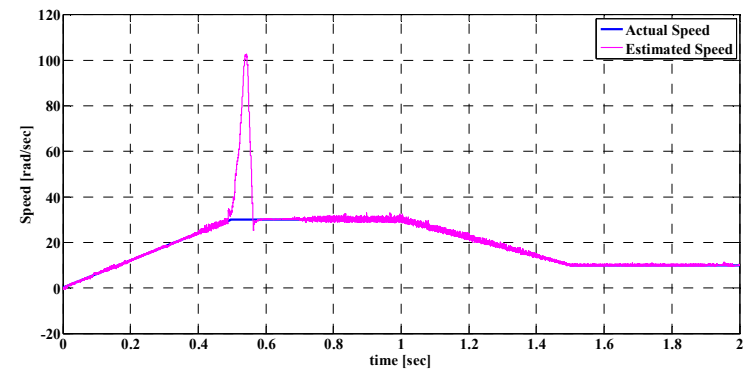

(a)

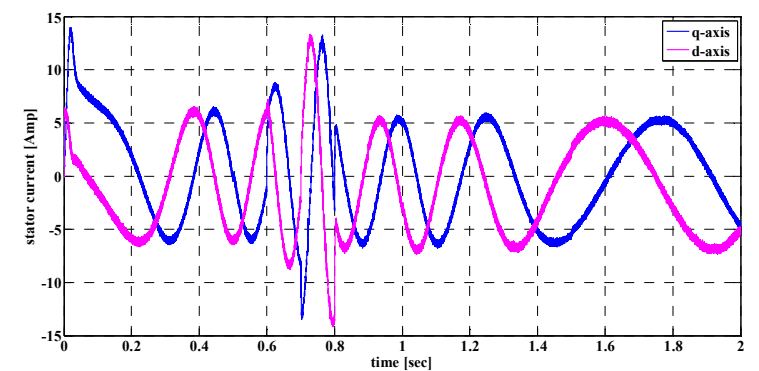

(b)

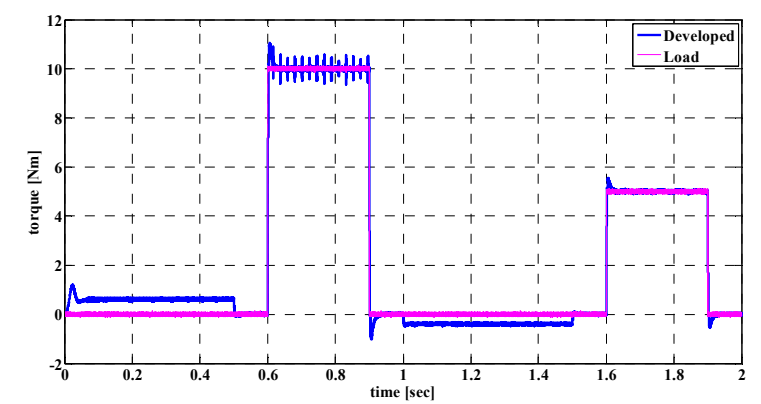

(c)

Fig. 4. Simulation results in case1: (a) speed [rad/sec], (b) d- and q- axis stator currents $[\mathrm{A}]$, (c) developed and load torque [Nm]

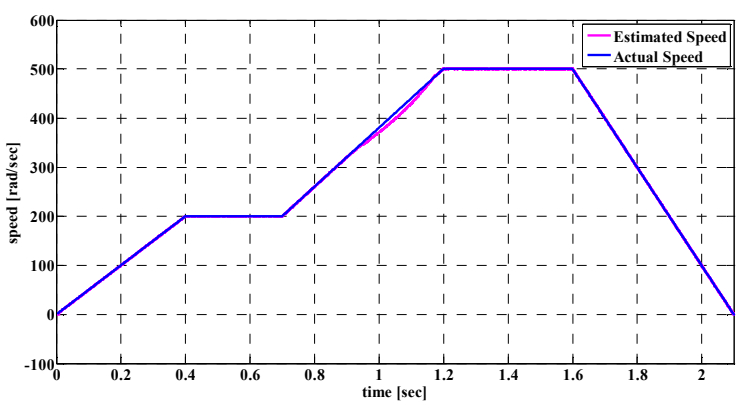

(a)

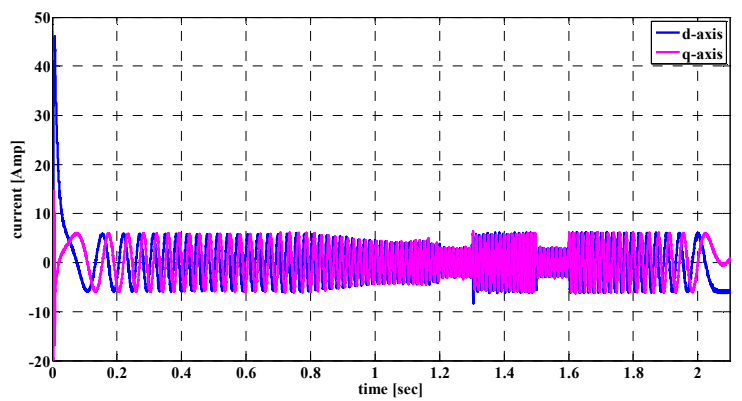

(b)

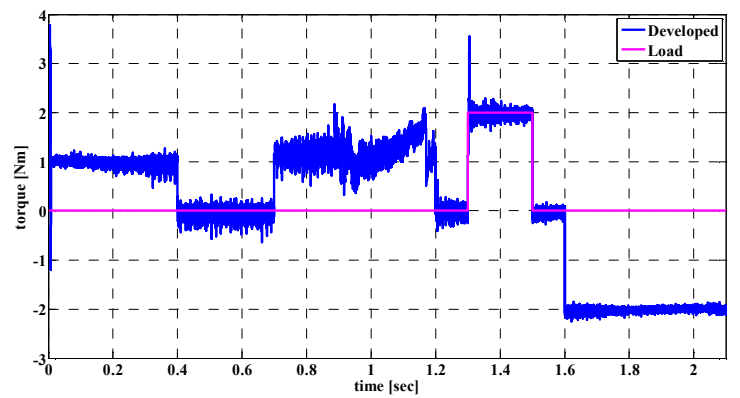

(c)

Fig. 5. Simulation results in case2: (a) speed [rad/sec], (b) d- and q- axis stator currents [A], (c) developed and load torque [Nm]

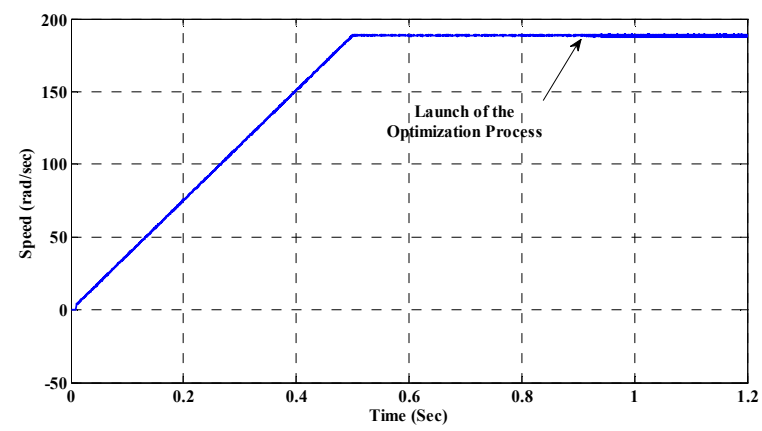

(a)

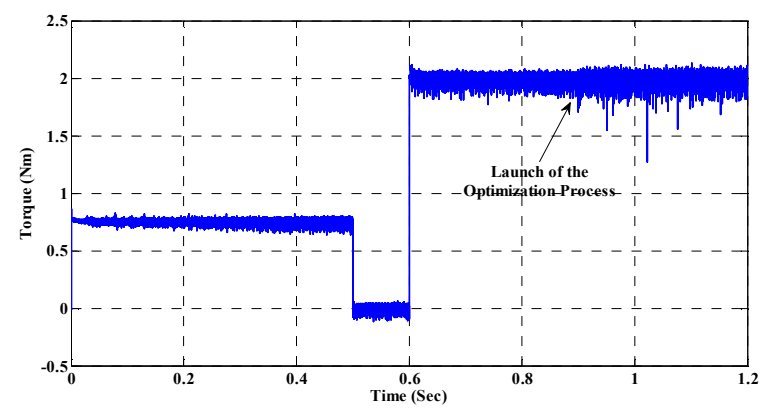

(b) 


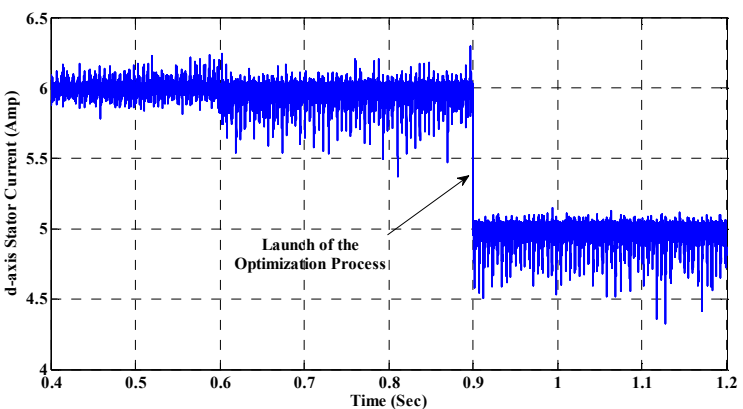

(c)

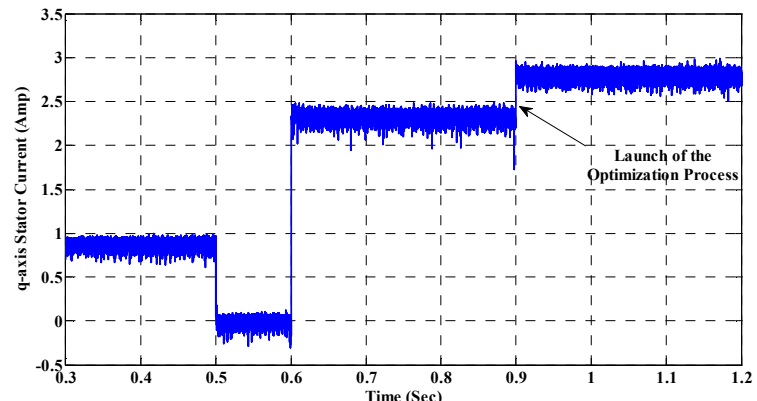

(d)

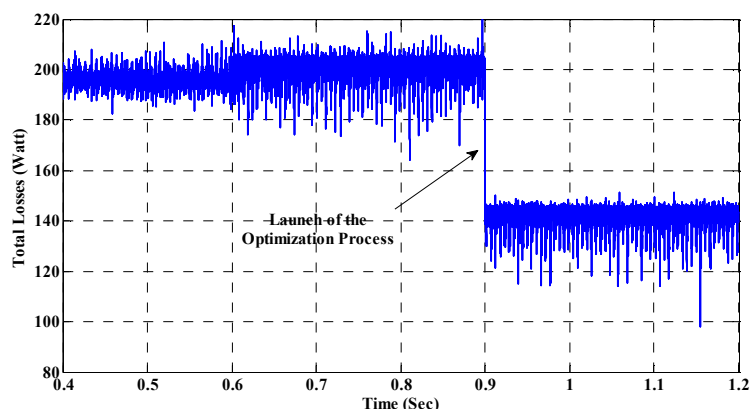

(e)

Fig. 6. Simulation results in case3: (a) speed [rad/sec], (b) developed torque [Nm], (c) d- axis stator currents [A], (d) and q- axis stator current [A], (e) total losses [w]

\section{CONCLUSION}

A novel speed-sensorless hybrid field-oriented and directtorque-controlled induction motor drive for electric vehicles has been presented. Simulation results above and below the rated speed show that the drive can fulfill all the requirements for electric vehicles. The extended Kalman filter was used for speed-sensorless motor control. It provides good speed estimates with speed estimation error of the order of a few revolutions per minute. The speed response meets the requirements of practical electric vehicles.

The effects of parameter uncertainty on speed estimation error and drive performance were also studied. A control strategy for robust field weakening operation of the induction motor drive was adopted to mitigate the effects of parameter variations. The efficiency of the drive system was improved by optimizing the flux during steady-state motor operation.

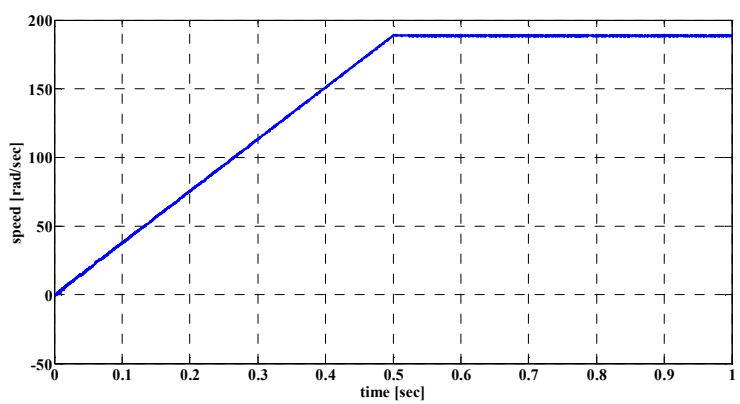

(a)

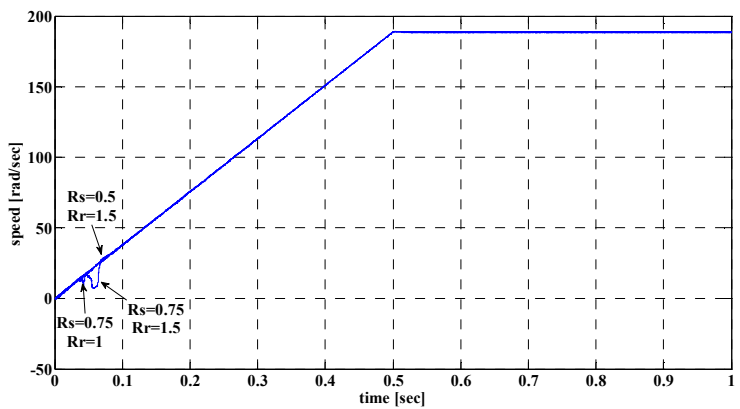

(b)

Fig. 7. Simulation results in case4: (a) estimated speed $[\mathrm{rad} / \mathrm{sec}]$ with correct parameters, (b) estimated speed [rad/sec] with incorrect parameters

\section{REFERENCES}

[1] C. C. Chan, A. Bouscayrol, K. Chen, "Electric, Hybrid, and fuel-cell vehicles: architectures and modeling," IEEE Trans. Veh. Technol., vol. 59, no. 2, pp. 589-598, Feb. 2010.

[2] M. Zeraoulia, M. E. H. Benbouzid and D. Diallo, "Electric motor drive selection issues for HEV propulsion systems: A comparative study," IEEE Trans. Veh. Technol., vol. 55, no. 6, pp. 1756-1764, Nov. 2006.

[3] D. Casadei, F. Profumo, G. Serra and A. Tani, "FOC and DTC: Two viable schemes for induction motors torque control," IEEE Trans. Power Elec. vol. 17, no. 5, pp. 779-787, Sep. 2002.

[4] S. Vaez-Zadeh, E. Jalali, "Combined vector control and direct torque control method for high performance induction motor drives," Elsevier Journal of Energy Conversion and Management, vol. 48, no. 5, pp. 3095-3101, May. 2007.

[5] M. Barut, S. Bogosyan, and M. Gokasan, "Experimental evaluation of Braided EKF for sensorless control of induction motors," IEEE Trans. Ind. Electron., vol. 55, no. 2, pp. 620-632, Feb. 2008.

[6] D. Casadei, G. Serra, A. Stefani, A. Tani and L. Zarri, "DTC drives for wide speed range applications using a robust field-weakening algorithm," IEEE Trans. Ind. Appl., vol. 54, no. 5, pp. 2451-2461, Oct. 2007.

[7] A. M. Bazzi, P. T. Krein, "Comparative evaluation of machines for electric and hybrid vehicles based on dynamic operation and loss minimization," Energy Conversion Congress and Exposition (ECCE) 2010, pp. 3345-3351.

[8] S.Lim, K. Nam, "Loss-minimising control scheme for induction motors," IEE Proc.-Electr. Power Appl., vol. 151, no. 4, July 2004.

[9] F. Abrahamsen, F. Blaabjerg, J. K. Pederson and P. B. Thoegersen, "Efficiency-optimized control of medium-size induction motor drives," IEEE Trans. Ind. Appl., vol. 37, no. 6, pp. 1761-1767, Nov/Dec. 2001

[10] M. Farasat, E. Karaman, "Efficiency-optimized hybrid field oriented and direct torque control of induction motor drive," International Conference on Electrical Machines and Systems (ICEMS) 2011, pp. 14. 